\title{
Fatigue assessment of semi-circular notched specimens produced by Laser Engineered Net Shaping method
}

\author{
Seyed Mohammad Javad Razavi ${ }^{1, *}$, Giancarlo Bordonaro ${ }^{2}$, Paolo Ferro $^{3}$, and Filippo \\ Berto $^{1}$ \\ ${ }^{1}$ Department of Mechanical and Industrial Engineering, Norwegian University of Science and \\ Technology (NTNU), 7491 Trondheim, Norway. \\ ${ }^{2}$ Institute for Mechanical Engineering and Materials Technology, University of Applied Sciences of \\ Southern Switzerland, CH-6928 Manno, Switzerland. \\ ${ }^{3}$ Department of Engineering and Management, University of Padova, Stradella San Nicola 3, 36100 \\ Vicenza, Italy.
}

\begin{abstract}
As a primary failure mechanism, fatigue failure of additively manufactured metallic parts has attracted considerable attention in both aerospace and biomedical applications. According to some specific design requirements, the majority of engineering components and structures contain notches of different shapes. Due to the intensive stresses around the notches, they are prone to crack nucleation. In this research, LENS (Laser Engineered Net Shaping) technology is used to fabricate Ti-6Al-4V semi-circular notched specimens. The fatigue analyses were performed to contribute to the fundamental understanding of the mechanical/cracking behaviour of additively manufactured LENS Ti-6Al-4V specimens weakened by semi-circular notches and subject to fatigue loading.
\end{abstract}

\section{Introduction}

Additive Manufacturing (AM) technologies allow the direct conversion of digital designs into physical products with direct production leading to avoid the setup time and the use of tools. A significant problem with this type of production stand up in failure assessment. The material properties of AM components change depending on the fabrication process making it to be dependent on the geometry of the components. This means that every change in the geometry will change the way that the AM machine performs its fabrication routine affecting the properties of the resulting solid. The AM components are no longer isotropic, commonly not fully dense, the surfaces roughness is considerable and there is a high risk of inclusions, inhomogeneities and impurities, all related to the underlying manufacturing strategy, which, in turn, is a function of the input geometry [1].

Fatigue has been considered as the primary failure mechanism in aerospace and biomedical applications for the components such as turbine blade, hip prothesis and mechanical heart valve. This fact led to high interest of industries in fatigue assessment of AM components. Leuders et al. [6] evaluated the fatigue behaviour of AM Ti-6Al-4V specimens and reported that the effect of porosities in the AM components on the fatigue behaviour is considerably 
more important that the effect of microstructure. In another research, Kasperovich and Hausmann [7] revealed that the reduction in fatigue strength of AM components is related to both microstructure of the AM material and also presence of defects. According to their experimental analyses, the martensite microstructure, lack of fusion between the layers, unmolten particles and microcracks were the sources for lower fatigue strength of AM parts. As a solution for this problem, they suggested application of Hot Isostatic Pressing (HIP), which reduces porosity, and they suggested machining the outer surface of the AM components to reduce the surface roughness. However, it is worth mentioning that due to complex geometry of some AM components, surface machining is not always possible. In this case "as-built" components should be directly used, and the surface roughness should be considered in fatigue evaluations.

The components designed by topology optimization and also orthopaedic implant usually contain geometrical discontinuities such as notches which are the sources for higher stress gradient and a potential location for fatigue crack initiation. The material behaviour around these notches is expected to be different from the rest of the components. Additionally, the surface condition and also presence of defects in this region can be different from the rest of AM components with more uniform geometry. Thus, it is necessary to pay attention to these notches, because they effect the fatigue resistance of component.

Currently, there are few researches conducted on the effect of geometrical discontinuities on the fatigue behaviour of AM parts. Hence, it is a very strategic point to fill this knowledge gap allowing future applicants to take full advantage of the unique features of AM, which will be key to integrate AM in every-day manufacturing. Both in aerospace and biomedical applications, fatigue is the primary mechanism of rupture in components such as turbine blade, hip prosthesis and mechanical heart valve [4-6]. Hence, the fatigue strength of AM parts has attracted considerable attention both in academic and industrial fields. For example, Razavi et al. [7] found that porosity acts as strong stress raiser and lead to brittle failure of LENS TiAl6V4 samples. Kasperovich and Hausmann [8] found a reduction in fatigue strength of SLM processed TiAl6V4 compared to the wrought alloy due to a combination of the unfavourable martensitic microstructure, unmolten particles, pores, and microcracks. Razavi et al. [9,10] studied the fatigue behaviour of notched components produced by Selective Laser Melting (SLM) method. They reported limited notch sensitivity of the AM parts due to presence of internal defects and rough surface.

this paper aims to contribute to the fundamental understanding of the fatigue behaviour of LENS Ti-6Al-4V specimens weakened by semi-circular notches. For this aim, the fatigue experiments were conducted on notched AM samples and the results are compared with that of smooth samples. In the first part of the paper, details of the manufacturing method that was used for AM fabrication of the samples are described. Afterwards, the fatigue results of notched samples are presented and compared.

\section{Experimental procedure}

The Ti-6Al-4V samples were manufactured by means of LENS by using optimized process parameters that guaranteed a high-density end product. An OPTOMEC LENS ${ }^{\mathrm{TM}}$ 850-R machine is used to fabricate flat Ti-6Al-4V samples in batches of one sample per substrate (Ti-6Al-4V, 6mm thick) and are deposited to a height of $81 \mathrm{~mm}$. Spherical Ti-6Al-4V Grade 5 powder with a mesh size of $-100 /+325$ (SAE AMS 4998C) is used for fabrication of testing samples. The processing parameters to have the minimum interlayer porosity were selected as $325 \mathrm{~W}$ laser power, $25 \mathrm{in} / \mathrm{min}$ head write speed, 0.02 in layer height, $0.015 \mathrm{in}$ hatch spacing, and $1.9 \mathrm{gr} / \mathrm{min}$ powder flow rate. Samples are fabricated with a $4 \times 16 \mathrm{~mm}$ 
cross-section and a length of $81 \mathrm{~mm}$ deposited over a $6 \mathrm{~mm}$ thick substrate (see Figure 1). All the LENS Ti-6Al-4V specimens were submitted to a stress relief annealing heat treatment for one hour into a preheated furnace at $600{ }^{\circ} \mathrm{C}\left(\sim 1100{ }^{\circ} \mathrm{F}\right)$ with a following cooling at room temperature. Afterwards, the testing samples were post-machined using a milling machine to fabricate the final geometry of the samples according to ASTM E 60692 standard (See Figure 2). The semi-circular notched specimens had stress concentration value of 1.308. The prepared samples had a mean roughness of $0.25 \mu \mathrm{m}$.

(a)

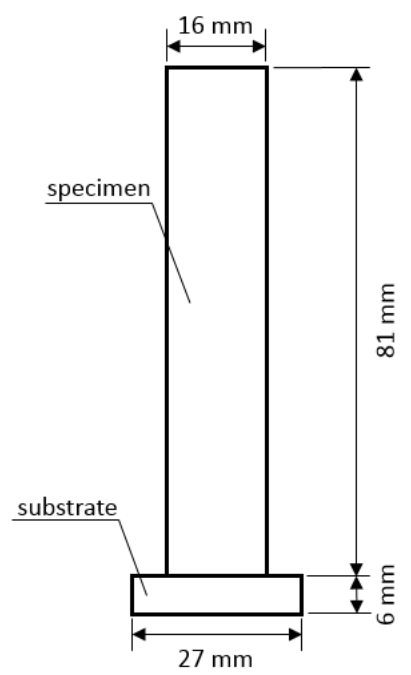

(b)

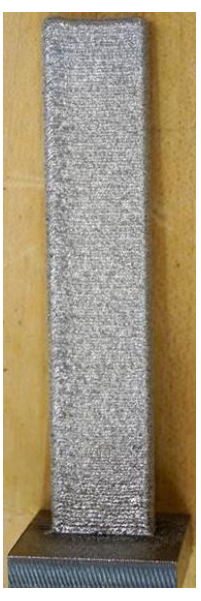

Fig. 1. (a) Schematic view of the initial block produced by LENS, and (b) as-built LENS Ti-6Al-4V sample deposited on the substrate.
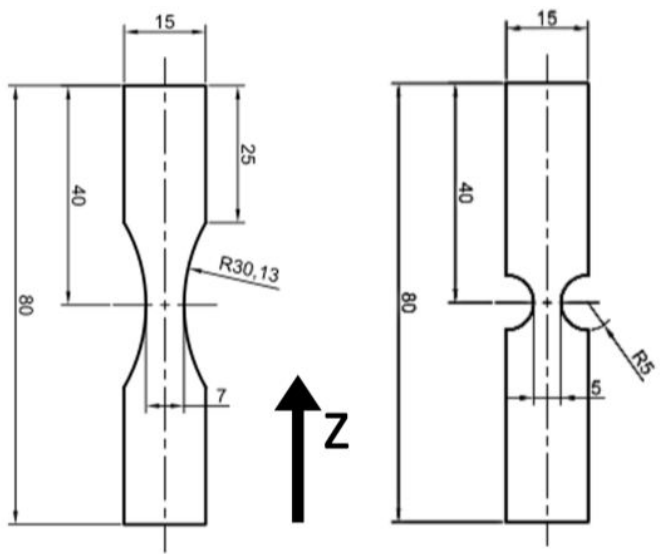

Fig. 2. Geometries of smooth and notched specimens and built axis ( $\mathrm{Z}$ ) (dimensions in $\mathrm{mm}$ ). 


\section{Results and discussion}

Figure 3 illustrates the results obtained from the statistical elaboration of fatigue test data for smooth and notched samples. Values of stress amplitude related to a survival probability of $50 \%$, the slope of the Wöhler curve, and the scatter index $\mathrm{T}_{\sigma}$, which is the ratio between the stress amplitudes corresponding to $10 \%$ and $90 \%$ of survival probability, are reported in Figure 3. Fatigue lives higher than 1 million are considered as run out and marked up with an arrow. It can be noted that the difference between the Wöhler curves are related only to the invers slope, but not to the mean value of the stress amplitude at $1 \times 10^{6}$ cycles and also the scatter index $T_{\sigma}$. The fatigue strength of both semi-circular notched specimens and smooth specimens at $10^{6}$ cycles was $477 \mathrm{MPa}$.

The notch sensitivity of the materials can be calculated using Equation (1):

$$
q=\frac{K_{f}-1}{K_{t}-1}
$$

in which $K_{f}$ is the fatigue notch factor $\left(K_{f}=\Delta \sigma_{A}^{\text {smooth }} / \Delta \sigma_{A}^{\text {nothed }}\right)$ and $K_{t}$ is the stress concentration factor defined by maximum local stress to nominal stress ratio $\left(K_{t}=\sigma_{\max } / \Delta \sigma_{\text {nom }}\right.$ ). The notch sensitivity factor, $\mathrm{q}$ has different values between zero to one for materials with no notch sensitivity to highly notch sensitive materials. According to the fatigue data, the notch factor has a value equal to one, which gives the notch sensitivity factor equal to zero. This can be related to the presence of possible material defects in the samples and in this case the failure of the material is governed by the interior defects and not by the notch geometries which were machined from the initial geometry produced by LENS.

Considering the notch sensitivity of the Ti-6Al-4V alloy, low notch sensitivity was also reported for the wrought material. Hoseini [11] reported that the presence of Hexagonal Close Packed (HCP) structure in alfa phase of the Ti-6Al-4V alloy results in lower notch sensitivity of this material. It is worth mentioning that the slip planes in HCP crystal lattice are all parallel to each other, a condition which is different from the material with Face Central Cubic (FCC) or Body Central Cubic (BCC) lattices. The slip systems number for dislocations in a HCP lattice are thus very low if compared to those in a FCC and BCC lattices. Because of its few slip systems, for a HCP lattice, such unfavorable orientations are very few. In this situation, the possibility of occurrence of many potential damage initiation sites within the thinner part of a smooth specimen is higher than that of the notched specimen with smaller critical length making it possible that almost no damage initiation site occurs in the small region around the notch tip where the stress is near its peak value. Hence, considering the local notch stress, the notched member can have higher fatigue resistant compared to the smooth specimen. 


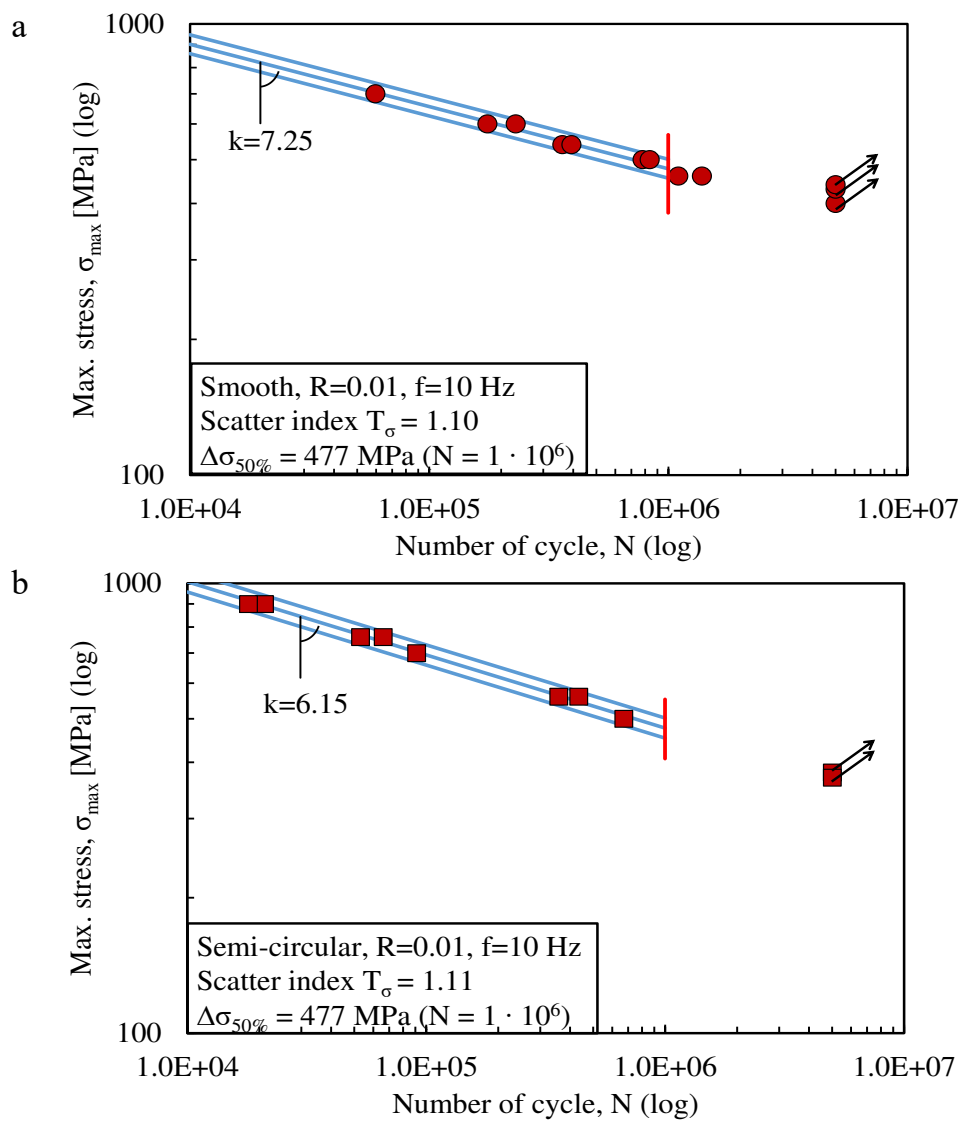

Fig. 3. Fatigue data from LENS additively manufactured specimens; (a) smooth samples, (b) semicircular samples.

The fracture surface of the tested samples was evaluated using Environmental Scanning Electron Microscopy (ESEM). According to ESEM results, fatigue cracks were observed to initiate from the internal defects such as lack of fusion or trapped voids as shown in Figure 4. The number of potential crack initiation sites in smooth samples is thought to be much higher than in the notched specimen where there is less possibility of existence of material defects in the smaller critical part of the AM samples near the notched region. The presence of these defects results in negligible notch sensitivity of the AM samples as observed by experiments. The fatigue crack initiates from one side or both sides of the tested samples and propagates towards the opposite edge covering almost the entire cross section of the sample until the final failure of the samples. The final rupture surface, characterized by dimples (Fig. 3c), was found at the centre or one side of the fracture surface for the smooth samples with two cracks on sides or one crack on a single side. The material defects were found to be close to the surface of the tested specimens and no porosity was observed on fracture surface of all samples showing the fact that presence of these defects can be related to poorer fabrication quality around the boundary of the LENS components. No significant porosity was observed in the inner part of the fracture surface. In a similar way, fatigue cracks nucleate at both the notch tip of the double semi-circular notched sample. 
a

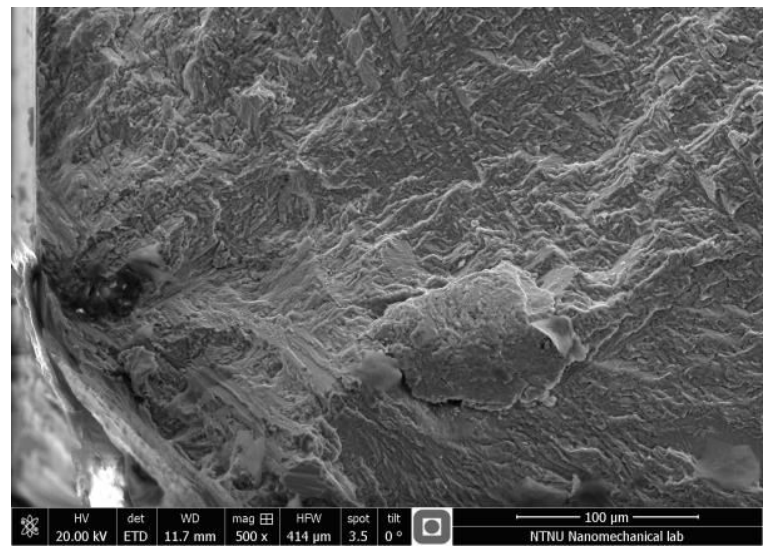

b

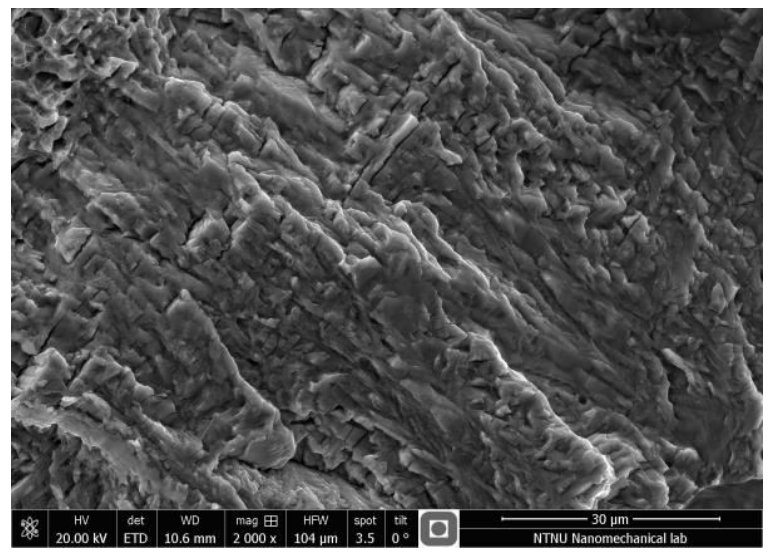

$\mathrm{c}$

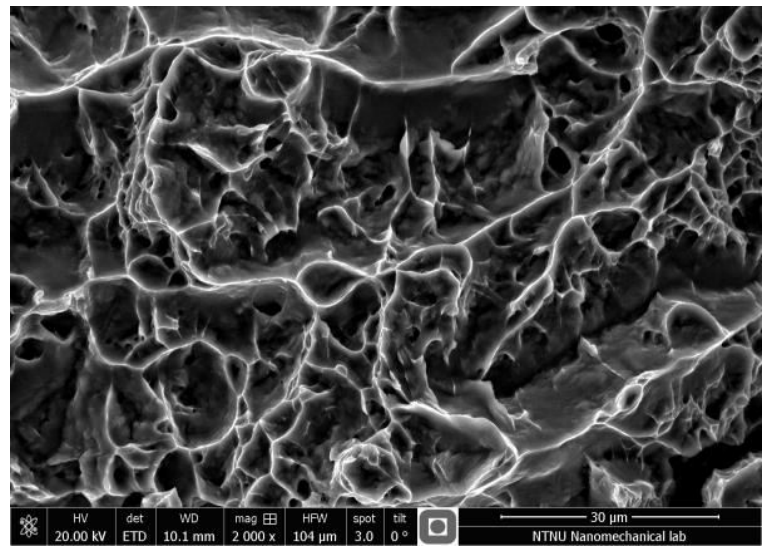

Fig. 4. ESEM picture taken from the fracture surface of the failed smooth specimen tested under fatigue loading; (a) fatigue crack initiation from a defect close to the surface of LENS notched specimen, (b) fatigue striation lines in the fatigue propagation part of the fracture surface, (c) deep dimples in the ductile rupture part of the fracture surface. 


\section{Conclusions}

The fatigue behaviour of Ti-6Al-4V semi-circular notched samples produced by LENS was experimentally investigated. The fatigue strength of notched specimens was compared with those corresponding to smooth samples and the fracture surface of the failed samples were analysed using ESEM to classify crack initiation points. Although the test specimens were weakened by the semi-circular notch, an identical fatigue strength was obtained for both notched and smooth specimens showing the low notch sensitivity of the material. This was attributed to the material defects close to the surface of the specimens, which acted as stress raisers.

\section{References}

1. F. Berto, S.M.J. Razavi, J. Torgersen, Frattura Integr. Strutt. 12(43), 1-32 (2018).

2. S. Leuders, M. Thöne, A. Riemer, T. Niendorf, T. Tröster, H.A. Richard, H.J. Maier, Int. J. Fatigue 48, 300-307 (2013).

3. G. Kasperovich, J. Hausmann, J. Mater. Process. Technol. 220, 202-214 (2015).

4. N.E. Cherolis, J. Fail. Anal. Preven. 8(3), 255-258 (2008).

5. X. Song, L. Wang, M. Niinomi, M. Nakai, Y. Liu, M. Zhu, Mater. Sci. Eng. A 619, 112-118 (2014).

6. Z. Sun, M. Chemkhi, P. Kanoute, D. Retraint, Mater. Sci. Eng. 63, 012021 (2014).

7. S.M.J. Razavi, G. Bordonaro, P. Ferro, J. Torgersen, F. Berto, Materials 11(2), 284 (2018).

8. G. Kasperovich, J. Hausmann, J. Mater. Process. Technol. 220, 202-214 (2015).

9. S.M.J. Razavi, P. Ferro, F. Berto, Metals, 7(8), 291 (2017).

10. S.M.J. Razavi, P. Ferro, F. Berto, J. Torgersen, Theor. Appl. Fract. Mech. (in press) (DOI: 10.1016/j.tafmec.2017.06.021)

11. S. Hosseini, Fatigue of Ti-6Al-4V. In Biomedical Engineering-Technical Applications in Medicine. InTech: Rijeka, Croatia, ISBN:978-953-51-0733-0 (2012). 